\title{
Susceptibilidad a la ciprofloxacina en Salmonella enterica serotipo Typhi, no multidrogorresistente, 2017 a 2020
}

\author{
DOI: $10.5377 /$ alerta.v4i3.10637 \\ José Eduardo Oliva Marín ${ }^{1^{*}}$, Esmeralda Villatoro ${ }^{2}$, Lucila Torres ${ }^{3}$, Miguel Oscar Grande ${ }^{4}$ \\ 1. Unidad de Investigación en Salud, Instituto Nacional de Salud/San Salvador, El Salvador. \\ 2, 3 y 4. Sección de Bacteriología, Laboratorio de Vigilancia en Salud Pública, Departamento de Laboratorio Nacional de Salud \\ Pública, Instituto Nacional de Salud, San Salvador, El Salvador \\ *Correspondencia \\ $\square$ joseduardoliva67@gmail.com \\ (1) 0000-0002-6005-0558
}

\section{G ACCESO ABIERTO}

Susceptibility to ciprofloxacin in nonmultidrug-resistant Salmonella enterica serotype Typhi, 2017 to 2020

Citación recomendada: Oliva Marín JE, Villatoro RE, Torres L, Grande MO. Susceptibilidad a la ciprofloxacina en Salmonella Typhi, no multidrogorresistente, 2017 a 2020. Alerta. 2021;4(3):170-175 DOI: 10.5377/alerta.v4i3.10637

Recibido:

12 de enero de 2021

Aceptado:

24 de mayo de 2021

Publicado:

26 de julio de 2021

\section{Contribución de autoría:}

JEOM': Elaboración del manuscrito en su totalidad con el apoyo de los coautores. EV': Elaboración de la sección de Resultados, junto con el autor principal. $\mathrm{LT}^{3}$ y MOG En el texto, elaboración de la sección de Metodología. Además, elaboración de las bases de datos de los aislamientos de Salmonella enterica Typhi en el DLNSP, durante el período de estudio establecido.

Conflicto de intereses:

Autores declaran no tener conflicto de interés.

\section{Resumen}

Introducción. Los patrones de susceptibilidad antimicrobiana de Salmonella enterica serotipo Typhi se han modificado globalmente durante las últimas tres décadas. Objetivo. Describir los patrones de susceptibilidad antimicrobiana de las cepas de Salmonella enterica Typhi, aisladas en El Salvador de enero 2017 a junio 2020. Metodología. Evaluación secundaria de las bases de datos del Laboratorio Nacional de Salud Pública, de los aislamientos de Salmonella enterica Typhi con sus respectivos antibiogramas, de muestras de pacientes que adolecieron de fiebre tifoidea en El Salvador, de enero 2017 a junio 2020. Resultados. 1406 aislamientos de Salmonella enterica Typhi fueron reportados. El $100 \%$ de los aislamientos analizados presentó susceptibilidad a la ceftriaxona y a la azitromicina. El 99,9\% de los aislamientos analizados presentó susceptibilidad a la ampicilina, al cloranfenicol, a la tetraciclina y al trimetoprim-sulfametoxazol. Para ciprofloxacina, se detectó susceptibilidad en el 8,5\% de las cepas analizadas, susceptibilidad intermedia en el 91,5\% y resistencia en el $0,08 \%$. Conclusión. Los hallazgos son compatibles con lo reportado a nivel mundial: el desarrollo rápido de susceptibilidad intermedia o resistencia a la ciprofloxacina, una vez esta es adoptada como el tratamiento de elección para la fiebre tifoidea. En El Salvador, los antibióticos antes considerados como de primera línea contra Salmonella enterica Typhi, deben ser reciclados.

Palabras clave

Salmonella enterica Typhi, farmacorresistencia microbiana, pruebas de sensibilidad microbiana, fluoroquinolonas.

\begin{abstract}
Introduction. The antimicrobial susceptibility patterns of Salmonella enterica serotype Typhi have changed globally during the last three decades. Objective. Describe the antimicrobial susceptibility patterns of Salmonella enterica Typhi isolates, registered in El Salvador from January 2017 to June 2020. Methods. A secondary evaluation of the National Public Health Laboratory Salmonella enterica Typhi databases was realized, with their respective antibiograms, using samples from patients who presented enteric fever in El Salvador, from January 2017 to June 2020. Results. 1406 Salmonella enterica Typhi isolates were reported. $100 \%$ of the analyzed isolates were susceptible to ceftriaxone and azithromycin. $99,9 \%$ of the analyzed isolates were susceptible to ampicillin, chloramphenicol, tetracycline and trimethoprim-sulfametoxazol. For ciprofloxacin, susceptibility was detected in $8,5 \%$ of the analyzed strains, intermediate susceptibility in $91,5 \%$ and resistance in $0,08 \%$. Conclusion. The findings are compatible with what has been reported worldwide: the rapid development of intermediate susceptibility or resistance to ciprofloxacin, once it's adopted as the treatment of choice for enteric fever. In El Salvador, antibiotics previously considered as first-line options against Salmonella enterica Typhi, must be recycled.
\end{abstract}

Keywords

Salmonella enterica Typhi, antimicrobial drug resistance, microbial sensitivity tests, fluoroquinolones.

\section{Introducción}

Las definiciones de susceptibilidad (S), susceptibilidad intermedia (I) y resistencia (R) han sido modificadas recientemente por EUCAST': al aislarse un microorganismo, este se clasifica en «susceptible» (término anterior: «sensible»), «susceptibilidad intermedia» o «resistente». Un microorganismo se considera susceptible cuando existe una alta probabilidad de éxito terapéutico utilizando la dosis estándar del antimicrobiano. 
Se considera que un microorganismo tiene susceptibilidad intermedia cuando existe una alta probabilidad de éxito terapéutico al incrementar la exposición al antibiótico, ya sea aumentando su dosis o por las concentraciones que este pueda alcanzar en el sitio de la infección. Un microorganismo se identifica como resistente cuando existe una alta posibilidad de falla terapéutica, incluso cuando hay una mayor exposición al antibiótico. La exposición es una función de la cual depende cómo: la vía de aplicación, la dosis, el intervalo, el tiempo de administración, la distribución y la excreción del antibiótico, influirán en el organismo infectante en el sitio de la infección.

En la década de 1960 iniciaron alrededor del mundo reportes de cepas de Salmonella enterica serotipo Typhi, en adelante S. Typhi, resistentes a los antibióticos de primera línea para su tratamiento: ampicilina, cloranfenicol o trimetoprim/sulfametoxazol (TMP/ SMZ). Se les denominó cepas clínicamente resistentes ${ }^{2}$; posteriormente, se les denominó cepas multidrogorresistentes (MDR), definiéndose como resistencia a tres o más clases de antimicrobianos. Estos reportes se hicieron cada vez más frecuentes en las décadas subsecuentes, conllevando a que las fluoroquinolonas (sobre todo la ciprofloxacina) se convirtieran en los antibióticos de elección para el tratamiento de la fiebre tifoidea, a partir de los años noventa ${ }^{3}$. Pocos meses después de este cambio, debido al uso excesivo y continuo de la ciprofloxacina, surgieron alrededor del mundo cepas con susceptibilidad intermedia o resistencia a este antimicrobiano ${ }^{4}$. El primer caso fue notificado por el Reino Unido en $1992^{5}$.

Actualmente, las cefalosporinas de tercera generación, consideradas como segunda alternativa, tanto en niños como en adultos, se están recomendando en Europa como fármacos de primera línea, condicionando el uso de la ciprofloxacina a la susceptibilidad mostrada en los antibiogramas 6 . Sin embargo, al igual que con la ciprofloxacina en la década de los noventa, han surgido cepas de S. Typhi resistentes a la ceftriaxona desde inicios del año 20004,7,8.

En América, para la fiebre tifoidea causada por S. Typhi MDR se recomienda iniciar el tratamiento con azitromicina o con una cefalosporina de tercera generación $n^{9,10}$. No obstante, en otras regiones del mundo ya se han reportado cepas de S. Typhi resistentes a la azitromicina ${ }^{4,8}$. Al igual que en Europa, la administración de ciprofloxacina queda como una opción alterna, dependiendo de la susceptibilidad local a dicho antibiótico.

Notablemente, en algunas regiones altamente endémicas del sur y del sureste de
Asia, las cepas de S. Typhi MDR están disminuyendo, emergiendo cada vez con más frecuencia cepas susceptibles a amoxicilina o a TMP/SMZ. Si la amoxicilina o la TMP/SMZ son considerados como opciones válidas, sobre la base de pruebas de susceptibilidad, un esquema de 14 días con cualquiera de los dos antibióticos es lo recomendado?.

En El Salvador, desde 2013 se reporta una elevada prevalencia de susceptibilidad intermedia a la ciprofloxacina en S. Typhi $(85 \%)^{11}$. Se describe el patrón de susceptibilidad antimicrobiana de S. Typhi en El Salvador, en el periodo de enero 2017 a junio 2020.

\section{Metodología}

Estudio descriptivo en el que se realizó una evaluación secundaria de las bases de datos del Laboratorio Nacional de Salud Pública (LNSP), de los aislamientos de S. Typhi con sus respectivos antibiogramas, de muestras de pacientes que adolecieron de fiebre tifoidea en El Salvador, en el periodo de enero de 2017 a junio de 2020.

Las cepas se analizan en el LNSP confirmando género, especie, serotipo y patrón de susceptibilidad antimicrobiana. Para el género y especie, se utilizan pruebas manuales (pruebas bioquímicas) y pruebas automatizadas (Vitek $2^{\circledR}$ Compact). Para su serotipificación se utilizó el esquema de KauffmannWhite $^{12}$. Las pruebas de susceptibilidad se efectúan con base al documento M100 Performance Standards for Antimicrobial Susceptibility Testing 2020, del Clinical and Laboratory Standards Institute ${ }^{13}$. Se evalúan los siguientes antimicrobianos: ampicilina, cloranfenicol, trimetoprim-sulfametoxazol, ceftriaxona, ciprofloxacina, ácido nalidíxico, tetraciclina y azitromicina.

Las bases de datos de S. Typhi del LNSP se reportan anualmente. Cada cepa se coloca en una fila y en las columnas se colocan las siguientes variables: registro de sección, edad y sexo del paciente, número de expediente clínico, departamento y municipio de origen, establecimiento de salud donde se tomó la muestra, fecha de recepción de la muestra, fecha de procesamiento de la muestra, semana epidemiológica, tipo de muestra, examen efectuado (cultivo bacteriológico), microorganismo identificado y antibiograma. La calidad de los datos se garantiza a través de protocolos establecidos por el LNSP.

Se integraron en una sola base de datos los aislamientos de S. Typhi reportados en El Salvador durante el periodo de estudio. Un total de 1406 aislamientos fueron registrados provenientes de los departamentos de Ahuachapán, Cabañas, Chalatenango, Cuscatlán, La Libertad, San Miguel, San Salvador, 
San Vicente, Santa Ana, Sonsonate y Usulután. No se recibieron muestras de tres de los catorce departamentos: La Paz, La Unión y Morazán. La base de datos se reorganizó de tal manera que incluyó para cada cepa las siguientes variables: registro de sección, departamento y municipio de origen, fecha de recepción de la muestra, fecha de procesamiento de la muestra, tipo de muestra, cultivo realizado, microorganismo identificado y antibiograma.

La susceptibilidad antimicrobiana no fue analizada en los 1406 aislamientos de S. Typhi reportados de enero de 2017 a junio de 2020, ya que no se contó con todos los antibióticos para tal fin. Lo anterior, debido a que los procesos de adquisición de estos antibióticos quedaron desiertos por problemas de oferta en el mercado. Por otro lado, con los antibióticos existentes, se priorizó el análisis de perfiles completos, inusuales y aquellos que procedían del Hospital Nacional de Niños Benjamín Bloom. La susceptibilidad fue analizada para ceftriaxona en 1382 (98\%), para ampicilina en 1364 (97\%), para trimetoprim-sulfametoxazol en 1355 (96\%), para tetraciclina en 1204 (85\%), para ciprofloxacina en 1194 (85\%), para ácido nalidíxico en 1108 (79\%), para cloranfenicol en 746 (53\%) y para azitromicina en 430 (30\%).

\section{Resultados}

Un total de 1406 aislamientos de S. Typhi fueron reportados en El Salvador de enero de 2017 a diciembre de 2020. San Salvador y La Libertad fueron los departamentos de donde se originó el $89 \%$ de las muestras, que representan el $81 \%$ y el $8 \%$ de estas, respectivamente. En cuanto a los munici- pios de origen de las muestras, los tres más frecuentes fueron Mejicanos (37,6\%), San Salvador $(33,5 \%)$ y Santa Tecla $(7,8 \%)$. Los primeros dos pertenecen al departamento de San Salvador, y Santa Tecla, al departamento de La Libertad.

Las muestras clínicas que fueron recibidas con mayor frecuencia fueron: sangre $(91,7 \%)$, heces (4\%) y médula ósea (2,2\%), que representan el $98 \%$ del total de las muestras.

De enero 2017 a junio 2020, el 100\% de los aislamientos analizados resultaron susceptibles a ceftriaxona y a azitromicina. En 2017, 1/746 aislamientos (0,13\%) presentó susceptibilidad intermedia a cloranfenicol. En 2018, 1/1204 aislamientos (0,08\%) presentó resistencia a tetraciclina. En 2019, $1 / 1364$ aislamientos $(0,07 \%)$ resulto resistente a ampicilina y en 2020, 1/1355 aislamientos $(0,07 \%)$ presentó susceptibilidad intermedia al trimetoprim-sulfametoxazol. De enero 2017 a junio 2020 se reportó resistencia al ácido nalidíxico en 1009/1108 (91\%) aislamientos, antibiótico que se utiliza para detectar de forma indirecta susceptibilidad intermedia a las fluoroquinolonas.

Para la ciprofloxacina, se detectó susceptibilidad [concentración inhibitoria mínima (CIM) de $\leq 0,06 \mu \mathrm{g} / \mathrm{mL}]$ en 101/1194 (8,5\%) aislamientos, susceptibilidad intermedia (CIM de 0,12 a $0,5 \mu \mathrm{g} / \mathrm{mL})$ en 1092/1194 (91,5\%) aislamientos y resistencia $(\mathrm{CIM} \geq 1,0 \mu \mathrm{g} / \mathrm{mL})$ en 1/1194 (0,08\%) aislamiento. Durante el periodo de estudio, la prevalencia de cepas de S. Typhi con susceptibilidad intermedia a la ciprofloxacina aumentó aproximadamente 10\% por año. En 2017 fue del 80\%, en 2018 del 90,7\% y en 2019 del 98,7\%, disminuyendo durante los primeros seis meses de $2020 \mathrm{a}$ 96,5\% (Figura 1).

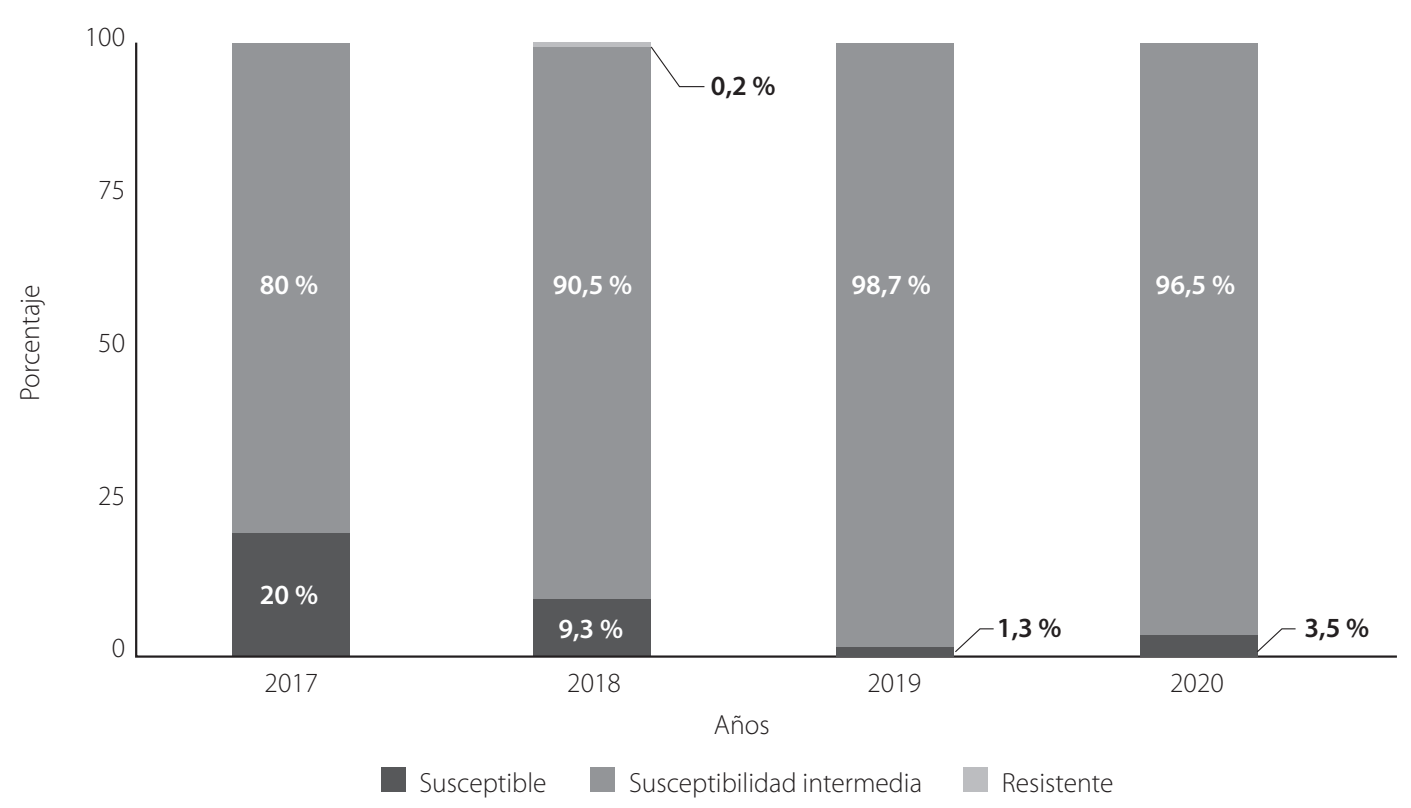

Figura 1. Susceptibilidad de S. Typhi a la ciprofloxacina en El Salvador, de enero de 2017 a junio de 2020 
Los patrones de susceptibilidad antimicrobiana de todas las cepas de S. Typhi, aisladas de enero de 2017 a junio de 2020, se muestran en la Tabla 1.

\section{Discusión}

El presente estudio reporta susceptibilidad intermedia a la ciprofloxacina en el 91,5\% de las cepas de S. Typhi analizadas en El Salvador, en el periodo de enero de 2017 a junio de 2020, con ausencia de resistencia a la ceftriaxona y a la azitromicina. Además, el 99,9\% de los aislamientos analizados presentó susceptibilidad a la ampicilina, al cloranfenicol, a la tetraciclina y al trimetoprimsulfametoxazol. El 91 \% reportó resistencia al ácido nalidíxico. La susceptibilidad al ácido nalidíxico ha sido validada como prueba de detección de susceptibilidad intermedia a la ciprofloxacina. La resistencia al ácido nalidíxico se asocia con CIM altas a la ciprofloxacina, lo cual puede conllevar a fracasos terapéuticos ${ }^{14}$.

Este patrón de susceptibilidad intermedia a la ciprofloxacina, con ausencia de MDR, ha sido reportado en otras regiones ${ }^{15}$. Sin embargo, esto no es lo usual, puesto que es la MDR el patrón predominante en países de ingresos bajos y medios ${ }^{16}$. Países que se caracterizan por no contar con acceso sostenible a agua potable o programas efectivos de saneamiento ambiental, sobre todo en áreas de gran densidad poblacional ${ }^{16}$. Lo anterior, podría explicar el mayor número de muestras provenientes de los municipios pertenecientes a los departamentos de San Salvador y la Libertad.

El porcentaje de cepas de S. Typhi que presentó susceptibilidad intermedia a la ciprofloxacina es superior a lo encontrado en otras regiones del mundo. Souza et al. ${ }^{17}$, de
1999 a 2006, reportaron 0\% (0/212) en el estado de Paraná, Brasil; Rahman et al. ${ }^{18}$, de 2002 a 2007, reportaron 47\% (307/654) en el Oriente Medio y Asia Central; Eibach et al. ${ }^{19}$, de 2007 a 2012, reportaron $53 \%$ (10/19) en Ghana; García et al. ${ }^{20}$, de 2008 a 2012, reportaron 24,2\% (8/33) en Perú; Al-Emran et al. ${ }^{21}$, en 2012, reportaron 19,5\% (8/41) en Kenia, y Alemán et al. ${ }^{11}$, en 2013, reportaron $85 \%$ (34/40) en El Salvador.

Se han descrito dos conductas que podrían explicar el surgimiento de la susceptibilidad intermedia y/o resistencia a la ciprofloxacina en S. Typhi. La primera, es el uso y abuso de la ciprofloxacina como antibiótico de "primera elección" contra múltiples procesos infecciosos, incluida la fiebre tifoidea ${ }^{15}$. La segunda, es el uso no terapéutico de la ciprofloxacina y otras fluoroquinolonas en la medicina veterinaria, como suplementos profilácticos o agentes promotores del crecimiento. Estos productos pueden facilitar la selección de bacterias resistentes o reducir la susceptibilidad a las fluoroquinolonas ${ }^{17}$.

Aunque la normativa salvadoreña ${ }^{22}$ coloca a la ciprofloxacina y a las cefalosporinas de tercera generación, como los fármacos de primera elección para el tratamiento de la fiebre tifoidea en adultos, los datos bacteriológicos locales indican que la ciprofloxacina no es una buena opción. Lo anterior, dada la alta prevalencia de susceptibilidad intermedia a este antibiótico identificado en el país.

Por lo anterior, aun cuando lineamientos establecidos sugieran el uso de ciertos antibióticos contra una patología específica, en este caso la fiebre tifoidea, la vigilancia rutinaria de los patrones de susceptibilidad a estos antibióticos es fundamental y debe ser tomada en cuenta para la decisión correcta del tratamiento a utilizar.

Tabla 1. Interpretación de antibiogramas de aislamientos de S. Typhi en El Salvador, de enero de 2017 a junio de $2020(n=1406)$

\begin{tabular}{lccccccccc|ccccccc}
\hline Año & \multicolumn{1}{c}{$\mathbf{2 0 1 7}$} & \multicolumn{1}{c}{$\mathbf{2 0 1 8}$} & \multicolumn{1}{c}{$\mathbf{2 0 1 9}$} & \multicolumn{3}{c}{ enero a junio 2020 } \\
\hline Susceptibilidad & $\mathrm{S}$ & $\mathrm{I}$ & $\mathrm{R}$ & $\mathrm{NA}$ & $\mathrm{S}$ & $\mathrm{I}$ & $\mathrm{R}$ & $\mathrm{NA}$ & $\mathrm{S}$ & $\mathrm{I}$ & $\mathrm{R}$ & $\mathrm{NA}$ & $\mathrm{S}$ & $\mathrm{I}$ & $\mathrm{R}$ & $\mathrm{NA}$ \\
\hline Tetraciclina & 189 & 0 & 0 & 46 & 422 & 0 & 1 & 35 & 393 & 0 & 0 & 121 & 199 & 0 & 0 & 0 \\
Ampicilina & 235 & 0 & 0 & 0 & 454 & 0 & 0 & 4 & 513 & 0 & 1 & 0 & 161 & 0 & 0 & 38 \\
Cloranfenicol & 39 & 1 & 0 & 195 & 447 & 0 & 0 & 11 & 60 & 0 & 0 & 454 & 199 & 0 & 0 & 0 \\
TMP/SMZ & 219 & 0 & 0 & 16 & 435 & 0 & 0 & 23 & 514 & 0 & 0 & 0 & 186 & 1 & 0 & 12 \\
Ciprofloxacina & 48 & 187 & 0 & 0 & 42 & 411 & 1 & 4 & 4 & 302 & 0 & 208 & 7 & 192 & 0 & 0 \\
Ácido nalidíxico & 48 & 0 & 182 & 5 & 43 & 0 & 411 & 4 & 4 & 0 & 302 & 208 & 4 & 0 & 114 & 81 \\
Cefoxitina & 235 & 0 & 0 & 0 & 449 & 0 & 0 & 9 & 375 & 0 & 0 & 139 & 106 & 0 & 0 & 93 \\
Ceftriaxona & 230 & 0 & 0 & 5 & 454 & 0 & 0 & 4 & 514 & 0 & 0 & 0 & 184 & 0 & 0 & 15 \\
Azitromicina & 32 & 0 & 0 & 203 & 341 & 0 & 0 & 117 & 57 & 0 & 0 & 457 & 0 & 0 & 0 & 199 \\
\hline
\end{tabular}

S: susceptible, I: susceptibilidad intermedia, R: resistente, NA: no analizado. 


\section{Conclusión}

En El Salvador se reporta una alta prevalencia de cepas de S. Typhi con susceptibilidad intermedia a la ciprofloxacina, por lo que este antibiótico no es idóneo como fármaco de primera elección.

Se reporta a la vez, susceptibilidad a los antimicrobianos antes considerados como de primera línea: ampicilina, cloranfenicol y TMP-SMZ, los cuales, además de la ceftriaxona y la azitromicina, actualmente son opciones para el tratamiento de la fiebre tifoidea en El Salvador.

\section{Agradecimiento}

Agradecimientos a licenciada María José Luna Boza, por su apoyo técnico durante la elaboración del manuscrito.

\section{Financiamiento}

Los autores declaran no tener fuente de financiamiento.

\section{Referencias bibliográficas}

1. EUCAST. New definitions of $S, I$ and $R$ from 2019. European Committee of Antimicrobial Susceptibility Testing. 2019. Fecha de consulta: 4 de marzo de 2021. Disponible en: https://www.eucast.org/newsiandr/

2. Smith SM, Palumbo PE, Edelson PJ. Salmonella strains resistant to multiple antibiotics: therapeutic implications. J Pediatric Infect Dis Soc. 1984;3(5):455-60. DOI: 10.1097/00006454-198409000-00017

3. Rowe B, Ward LR, Threlfall EJ. Treatment of multiresistant typhoid fever. The Lancet. 1991;337(8754):1422. DOI: 10.1016/01406736(91)93116-Q

4. Kirchhelle C, Dyson ZA, Gordon DA. Biohistorical perspective of typhoid and antimicrobial resistance. Clin Infect Dis. 2019;69(Suppl 5):S388-S394. DOI: 10.1093/ cid/ciz556.

5. Umasankar S, Wall RA, Berger J. A case of ciprofloxacin-resistant typhoid fever. Commun Dis Rep CDR Rev. 1992;2(12):R13940. PMID: 1284936

6. Trawinski H, Wendt S, Lippmann N, Heinitz S, von Braun A, Lübbert C. Typhus abdominalis und Paratyphus. Z Gastroenterol. 2020;58(2):160-170. DOl: 10.1055/a-1063-1945

7. Yousafzai MT, Qamar FN, Shakoor S, Saleem K, Lohana H, Karim S, et al. Ceftriaxoneresistant Salmonella Typhi outbreak in Hyderabad city of Sindh, Pakistan: high time for the introduction of typhoid conjugate vaccine. Clin Infect Dis. 2019;68(Suppl 1):S16-S21. DOI: 10.1093/cid/ciy877

8. Marchello CS, Carr SD, Crump JA. A systematic review on antimicrobial resistance among Salmonella Typhi worldwide. Am JTrop Med Hyg. 2020 Dec;103(6):2518-2527. DOI: 10.4269/ ajtmh.20-0258.

9. American Academy of Pediatrics. Salmonella infections In: Kimberlin DW, Brady MT, Jackson MA, Long SS, eds. Red Book: 2018 Report of the Committee on Infectious Diseases. 31st ed. Itasca, IL: American Academy of Pediatrics; 2018:711-718.

10. Crump JA, Sjölund-Karlsson M, Gordon MA, Parry CM. Epidemiology, clinical presentation, laboratory diagnosis, antimicrobial resistance, and antimicrobial management of invasive Salmonella infections. Clin Microbiol Rev. 2015;28(4):901-37. DOI: 10.1128/ CMR.00002-15

11. Alemán R. Ávalos B, Argueta C. Determinación de la susceptibilidad a la ciprofloxacina en cepas de Salmonella Typhi, en el cepario del Laboratorio Nacional de Referencia, por el método epsilométrico (E-test) durante el período de julio a septiembre de 2013. Universidad de EI Salvador; 2013. Disponible en: http://ri.ues. edu.sv/id/eprint/7407/1/50108070.pdf

12. WHO Collaborating Centre for Reference and Research on Salmonella. Antigenic formulae of the Salmonella serovars. WHO/ Institut Pasteur. 2007. Consultado el 5 de marzo de 2021. Disponible en: https://www. pasteur.fr/sites/default/files/veng 0.pdf

13. CLSI. Performance Standards for Antimicrobial Susceptibility Testing. 30th ed. CLSI supplement M100. Clinical and Laboratory Standards Institute, 950 West Valley Road, Suite 2500, Wayne, Pennsylvania, 19087, USA, 2020;40(1). Disponible en: https://clsi.org/media/3481/ m100ed30 sample.pdf

14. Bhagra S, Sood A, Singh D, Kanga A. Increased resistance to nalidixic acid and ciprofloxacin in Salmonella isolates from the Sub Himalayan region. Int J Res Med Sci. 2017;5(9):4025. DOl: 10.18203/2320-6012. ijrms20173976

15. Misra R, Prasad KN, Amrin N, Kapoor P, Singh $\mathrm{S}$, Ghar M. Absence of multidrug resistance in Salmonella enterica serotypes Typhi and Paratyphi A isolates with intermediate susceptibility to ciprofloxacin. Trans R Soc Trop Med Hyg. 2015;109(8):538-40. DOI: 10.1093/trstmh/trv036

16. Browne AJ, Kashef Hamadani BH, Kumaran E, et al. Drug-resistant enteric fever worldwide, 1990 to 2018: a systematic review and meta-analysis. BMC Med. 2020;18(1):1. DOl: 10.1186/s12916-019-1443-1 
17. Souza RB, Ferrari RG, Magnani M, Kottwitz LB, Alcocer I, Tognim MC, et al. Ciprofloxacin susceptibility reduction of Salmonella strains isolated from outbreaks. Braz J Microbiol. 2010;41(2):497-500. DOI: 10.1590/S1517838220100002000033

18. Rahman BA, Wasfy MO, Maksoud MA, Hanna N, Dueger E, House B. Multi-drug resistance and reduced susceptibility to ciprofloxacin among Salmonella enterica serovar Typhi isolates from the Middle East and Central Asia. New Microbes New Infect. 2014;2(4):88-92. DOI: 10.1002/nmi2.46

19. Eibach D, Al-Emran HM, Dekker DM, Krumkamp R, Adu-Sarkodie Y, Cruz Espinoza $L M$, et al. The emergence of reduced ciprofloxacin susceptibility in Salmonella enterica causing bloodstream infections in rural Ghana. Clin Infect Dis. 2016;62(Suppl 1):S32-6. DOI: $10.1093 /$ cid/civ757
20. García C, Lejon V, Horna G, Astocondor L, Vanhoof R, Bertrand S, et al. Intermediate susceptibility to ciprofloxacin among Salmonella enterica serovar Typhi isolates in Lima, Perú. J Clin Microbiol. 2014;52(3):96870. DOI: 10.1128/JCM.02663-13

21. Al-Emran HM, Eibach D, Krumkamp R, Ali M, Baker S, Biggs HM, et al. A multicountry molecular analysis of Salmonella enterica serovar Typhi with reduced susceptibility to ciprofloxacin in Sub-Saharan Africa. Clin Infect Dis. 2016;62(Suppl 1):S42-6. DOI: 10.1093/cid/civ788

22. Minsal. Guías clínicas de medicina interna. Ministerio de Salud de El Salvador. 2018. Disponible en: http://asp.salud.gob. sv/regulacion/pdf/guia/guia clinica medicina interna $\vee 1 . p d f$ 